\title{
Diplomacia económica: Aproximaciones conceptuales y su aplicación en la política de Xi Jinping hacia el Sur Global
}

Florencia Rubiolo*
Virginia S. Busilli**

\section{RESUMEN}

La economía política internacional (EPI) se focaliza en precisar cómo las estructuras de intereses, poder y finanzas afectan las relaciones económicas entre Estados. Una corriente más reciente de esa literatura se ocupa de la diplomacia económica. El diferencial de este concepto radica en la utilización de instrumentos económicos para perseguir objetivos políticos. Desde finales de los noventa, y con mayor impulso a partir de la crisis de 2008, el ascenso económico y la proyección internacional de China están acompañados por una mayor disposición del uso de herramientas de diplomacia económica en función de objetivos internos y externos. La Iniciativa de la Franja y la Ruta (BRI) es un claro ejemplo de esta articulación, con especial incidencia en las regiones del Sur Global. Este estudio analiza los conceptos vinculados a diplomacia económica para luego profundizar en su articulación con la política exterior de Xi Jinping, y su proyección hacia el Sur Global. Concluye que Beijing intenta desarrollar y proyectar su poder económico a través del BRI, tanto en su periferia, como en el resto del globo. Asimismo, también responde a objetivos de naturaleza doméstica, vinculado a su proceso modernizador.

* Doctora en relaciones internacionales. Investigadora. Ciecs-Conicet/Universidad Siglo 21, Córdoba (Argentina). [frubiolo@gmail.com]; [https://orcid.org/0000-0002-5669-7332].

** Doctoranda en estudios sociales de América Latina. Ciecs-Conicet/Universidad Católica de Córdoba, Córdoba (Argentina). [virginia.busilli@gmail.com]; [https://orcid.org/0000-0002-6305-8010].

Recibido: 30 de noviembre de 2020 / Modificado: 15 de febrero de 2021 / Aceptado: 22 de febrero de 2021

Para citar este artículo:

Rubiolo, F. y Busilli, V. S. (2021). Diplomacia económica: Aproximaciones conceptuales y su aplicación en la política de Xi Jinping hacia el Sur Global. OASIS, 34, pp. 127-150

DOI: https://doi.org/10.18601/16577558.n34.08 
Palabras clave: Diplomacia económica, Iniciativa de la Franja y la Ruta, asimetría económica, política exterior china

\section{ECONOMIC DIPLOMACY: CONCEPTUAL APPROACHES AND APPLICATION TO XI JINPING'S POLICY IN THE GLOBAL SOUTH}

\section{ABSTRACT}

International Political Economy (IPE) focuses on how interest, power and finance structures control economic relations between states. A more recent field of that literature deals with economic diplomacy. The differential of this concept lies in the use of economic instruments to pursue political objectives. Since the end of the 1990s, and with greater momentum since the 2008 crisis, China's economic rise and international projection have been accompanied by a greater willingness to use economic diplomacy tools based on internal and external goals. The Belt and Road Initiative (BRI) is a clear example of this process, with special incidence in the regions of the Global South. This study analyzes the concepts related to economic diplomacy and the interaction with the foreign policy of $\mathrm{Xi}$ Jinping, and its projection towards the Global South. It concludes that Beijing tries to develop and project its economic power through BRI, both in its periphery and around the globe. Likewise, it also responds to objectives of a domestic nature, linked to its modernization process.

Keywords: Economic Diplomacy, Belt and Road Initiative, economic asymmetry, Chinese foreign policy

\section{INTRODUCCIÓN}

Si una mariposa aletea en China, sacude al mundo. El 2020 fue una muestra contundente de esta metáfora, no solo por el impacto global de la pandemia, sino por la evidencia de la compleja y profunda interconexión económica entre los Estados. La República Popular China (RPC) comenzó en 1978 un proceso de apertura y modernización bajo la dirección de Deng Xiaoping, con la intención de llevar al país a una senda de desarrollo que, acercándose al capitalismo, permitiera mejorar las condiciones socioeconómicas de la mayor y una de las más empobrecidas poblaciones del mundo. Cuatro décadas más tarde, a un ritmo sin precedentes, se consolidó como segunda economía mundial y concentra el mayor flujo de comercio del globo. Como consecuencia, sus decisiones, internas y externas, tienen impactos globales.

La estrategia de crecimiento china se construyó sobre la combinación de una enorme masa de mano de obra barata, mercados internacionales e importación de materias primas, de tal modo que hoy su economía se encuentra íntimamente ligada y no puede ser separada de las de los demás países (Garlick, 2020). Esta conexión también implica que la economía, principal fuente de proyección del poder de China hoy, se puede convertir en una herramienta para la concreción de intereses políticos a través de la influencia en terceros Estados y actores internacionales.

En este debate, donde se entrecruzan las dimensiones económica y política, es que se sitúa el argumento del artículo. La economía política internacional, y dentro de ella los conceptos de diplomacia económica, incentivos e 
influencia, se entrelazan en la política exterior China y, en particular, en la relativamente nueva Iniciativa de la Franja y la Ruta. La diplomacia económica es un concepto neurálgico para comprender las dinámicas globales que reemergen con fuerza a principios de los noventa y adquiere mayor centralidad en el actual siglo, en paralelo con el debilitamiento de las instancias económicas multilaterales.

En este marco, la Iniciativa de la Franja y la Ruta es una compleja, multidimensional y flexible estrategia de alcance global ${ }^{1}$ de China que se convirtió en la herramienta central de la diplomacia económica del país. Emblema de la era de Xi Jinping, la BRI constituye un ambicioso plan de infraestructura y conectividad que está comenzando a reconfigurar la geoeconomía de los países en desarrollo, principalmente de los comprendidos en los corredores centrales. Partiendo de la revisión y actualización de los conceptos vinculados a diplomacia económica, este trabajo se propone abordar las principales características de la Iniciativa de la Franja y la Ruta, desde una mención particular de las motivaciones chinas (vinculadas a las dimensiones económicas y políticas). Finalmente, y como foco central del artículo, se abordan las principales implicancias de las inversiones que se comprenden dentro de la BRI en el Sur Global, y su vinculación con los intereses nacionales de
Beijing dentro de una estrategia de conversión de poder económico en poder político global.

\section{UNA APROXIMACIÓN TEÓRICA A LA DIPLOMACIA ECONÓMICA}

Definir diplomacia económica es una tarea de creciente complejidad, consecuencia de la multiplicidad de actores, niveles, procesos, instrumentos y resultados que forman parte de su creación y entramado. La interdependencia económica contribuye aún más a complejizar la definición, al desdibujar de manera incesante la frontera entre lo internacional y lo doméstico, generando dinámicas híbridas y multinivel (Bayne y Woolcock, 2018).

El fin de la guerra fría y el desmantelamiento de las posibilidades de enfrentamiento militar directo entre grandes potencias, abonaron el terreno para el surgimiento de nuevas lecturas respecto del devenir de la competencia internacional (Scholvin y Wigell, 2019). Los cambios en la estructura económica global, impulsados por los rápidos avances tecnológicos, tuvieron un impacto ineludible en la forma de entender la política internacional, el poder y la diplomacia (Strange, 1992). La competencia interestatal sufrió una irreversible traslación: del territorio a la riqueza. Es decir, la interdependencia entre Estados y entre empresas y Estados, pasó a ocupar el centro de la

\footnotetext{
Si bien la Iniciativa tiene como ejes principales los continentes europeo, asiático y africano, se encuentra abierta a cualquier país del mundo que desee participar. Este aspecto demuestra el carácter flexible antes mencionado, así como una puerta hacia la proyección global, que permite a China modificar, ajustar e incluso redefinir el alcance de la Iniciativa.
} 
escena como condicionante de las dinámicas económicas internacionales, favoreciendo la reproducción de vínculos multinivel y multiactor (Stopford y Strange, 1991). Se fortaleció una perspectiva transdisciplinaria, que adentra lo internacional en lo propiamente nacional y proyecta lo nacional a las pujas externas (Tussie, 2015).

En esta línea, Amin y Thrift (1997, pp. 148-149) sintetizan las implicancias de lo que denominan las influencias trans-territoriales: 1) creciente centralidad de la estructura financiera internacional, principal motor de la integración de la economía global; 2) la importancia de la estructura de conocimiento; 3) la internacionalización de la tecnología; 4) el surgimiento de oligopolios transnacionales $y$, finalmente, 5) en paralelo a la globalización de las finanzas, el conocimiento y la producción, hay un surgimiento de una diplomacia económica transnacional y una reorientación global de las estrategias estatales.

Además de la transformación en las formas de competencia interestatal, y de los actores involucrados, se suman a estas condiciones la traslación paulatina de poder de Occidente hacia el este de Asia -y también dentro de la región de Asia Pacífico- apuntalado por el fortalecimiento económico y político de China y su proyección internacional (OkanoHeijmans, 2013). En este sentido, entendemos que determinadas condiciones de la estructura internacional pueden funcionar como incentivos para la implementación de una diplomacia económica más activa (Mastanduno, 1998). Siguiendo a Coolsaet (2004, p. 61), "la diplomacia económica se fortalece cuando la aceleración de la globalización es acompa- ñada por una ausencia de reglas de conducta consensuadas y por la emergencia de nuevos temas en la agenda comercial como resultado de una revolución industrial". Este contexto sistémico, que plantea un incremento de la competencia en un escenario volátil e incierto, genera mayores incentivos a las empresas a buscar el apoyo de los Estados, así como a estos a procurar condiciones favorables a sus firmas. El resultado es la intensificación de la diplomacia económica, y el fortalecimiento del rol del Estado como actor central (Coolsaet, 2004; Okano-Heijmans, 2011), en un escenario internacional que presenta mayores amenazas y retos a los actores estatales y no estatales.

El concepto de diplomacia económica, en términos amplios, puede definirse como una sumatoria de procesos, prácticas e instrumentos mediante los cuales se crean y distribuyen los beneficios de las relaciones económicas internacionales (Woolcok y Bayne, 2018). Esta definición, por su amplitud, contempla todo tipo de acciones, prácticas, estrategias y decisiones de naturaleza económica emanadas hacia el exterior del Estado. Sin dudas, no todas las actividades económicas implican diplomacia, ni todas las acciones de actores económicos persiguen objetivos vinculados con los propósitos nacionales. El rol del Estado como actor primario (Okano-Heijmans, 2011; Hill, 2016), aunque no único, en estos procesos, contribuye a definir el carácter político vinculando la esfera de actividades económicas y sus actores con objetivos estatales.

En una definición más detallada, Rana (2013) plantea que la diplomacia económica "es el proceso a través del cual los países abordan la esfera económica externa para maxi- 
mizar sus ganancias nacionales en diversos campos, incluidos el comercio, la inversión y otras formas de intercambios económicamente beneficiosos, donde gozan de una ventaja comparativa”. En un sentido similar, Hill (2016, p. 161) subraya que "la diplomacia económica deriva de la necesidad particular de promover la prosperidad nacional y conducir una política exterior económica para lograrlo". OkanoHeijmans (2011, p. 17), agrega complejidad a la definición al postular que la diplomacia económica involucra "el uso de medios políticos como influencia en negociaciones internacionales, con el objetivo de aportar a la prosperidad nacional, así como el uso de medios de influencia económica para incrementar la estabilidad política de la nación”. Berridge y James (2003, p. 91) contribuyen en este sentido planteando que la diplomacia económica se involucra en cuestiones de política económica, como las instancias de negociaciones comerciales multilaterales, pero también incluye la utilización de recursos económicos, en forma de incentivos o sanciones, en procura de determinado objetivo de política exterior. En este último sentido, los autores se refieren a lo que Baldwin (1985) entiende como economic statecraft.

La diplomacia económica entonces, se desarrolla en el terreno de intersección de los objetivos, las estrategias y los actores políticos y económicos, que operan en el nivel internacional respondiendo a condicionamientos domésticos y externos. La naturaleza de las estrategias y objetivos puede tener una mayor preeminencia de lo económico o de lo político, y puede procurar condicionar o influir en el comportamiento de un tercer Estado -o incluso actores no estatales dentro de otros Estados- persiguiendo objetivos nacionales.

El debate en torno a los objetivos que se persiguen a través de la diplomacia económica y la eficacia de los instrumentos tienen un prominente recorrido. Desde una perspectiva economicista, el foco se centra en los instrumentos económicos que implementa el Estado a nivel internacional en la procura del bienestar y la prosperidad económica de la nación (Rana, 2013; Rabby, 2015). Estos estudios se concentran en la labor de las oficinas gubernamentales -vinculados a la acción externa- y su interacción con actores no estatales en el ámbito de las negociaciones económicas internacionales bilaterales, regionales y multilaterales (Saner y Yiu, 2003). La diplomacia comercial, la diplomacia financiera y la diplomacia empresarial se incluyen como vertientes dentro de esta visión.

Desde una perspectiva de economía política internacional, los objetivos y los instrumentos, económicos y políticos, adquieren una importancia equivalente como elementos de la diplomacia económica. Es decir, "implica una combinación de objetivos de política exterior y herramientas comerciales, y objetivos comerciales y herramientas políticas en un entorno determinado donde se configura o practica la diplomacia económica" (Okano-Heijmans, 2011 , p. 27). En una tercera perspectiva, desde una visión Estado-céntrica de las relaciones internacionales, más próxima a la corriente realista, la diplomacia económica acentúa el poder como elemento determinante. La preservación del Estado -seguridad- y la persecución de intereses nacionales definidos en términos de poder, se convierten en conceptos centrales desde esta visión. 
Una de las nociones más ampliamente desarrollada desde este enfoque es la de economic statecraft. Este concepto tiene como diferencial la utilización del poder económico -sea en forma de incentivos o de castigos/sancionespara modificar decisiones, comportamientos o acciones de terceros Estados, de manera que respondan a intereses políticos del Estado emisor. Baldwin (1985) es uno de los principales referentes en esta línea. De acuerdo con el autor, se puede considerar como economic statecraft toda política que tenga los siguientes tres componentes: el tipo de instrumento utilizado para influir es de naturaleza económica; el objetivo del intento de influencia es otro actor internacional (primordialmente otro Estado); el alcance de la medida es alguna dimensión del comportamiento de otro actor (Baldwin, 1985, p. 32).

Los instrumentos utilizados por los Estados para ejercer influencia sobre otros son múltiples, pero se clasifican en dos grandes grupos genéricos: sanciones e incentivos. Mientras las sanciones hacen referencia a medidas punitivas o amenazas, los incentivos se refieren a premios o recompensas. En este sentido, las medidas pueden tener una naturaleza negativa o positiva, pero incluso los incentivos se convierten en potenciales sanciones (Hirshman, 1980, en Blanchard y Ripsman, 2008). Retomamos aquí la afirmación de Gilpin (2001, p. 81) que postula que "los lazos económicos entre los Estados casi siempre involucran relaciones de poder", a través de la construcción de dependencias que son políticamente explotadas por el lado más fuerte. En consecuencia, los Estados tienen incentivos "para aumentar la dependencia de otros Estados a través de políticas tales como la ayuda extranjera y las concesiones comerciales" (Gilpin, 2001, p. 82). El presente trabajo parte de esta última perspectiva dado que, en el caso de la política económica de China a nivel internacional, la utilización de herramientas que influencian el comportamiento de terceros Estados comienza a observarse con mayor asiduidad desde 2008.

La crisis financiera y el relativo éxito con el que el pcch logró superar sus consecuencias a nivel interno, terminó consolidando al país como el motor de la recuperación económica global (Xiaotong y Keith, 2017). Así, la política de ascenso pacífico ya se había instalado como parte del discurso y las acciones de política exterior, que suma una nueva y ambiciosa estrategia: la Belt and Road Initiative. A través de esta estrategia económica China comienza a extender su alcance internacional, especialmente en la medida en que se ha aplicado a los países en desarrollo (Ferchen, 2018).

\section{BELT AND ROAD COMO HERRAMIENTA DE DIPLOMACIA ECONÓMICA CHINA}

La iniciativa se presentó por primera vez en septiembre de 2013, durante el discurso de Xi en la Universidad Nazarbayev de Kazajstán, donde propuso construir un cinturón económico de la ruta de la seda. En octubre de 2015, presentó la Ruta de la Seda Marítima del Siglo xxi durante su discurso en la Cámara de Representantes de Indonesia.

Inicialmente denominada como "Una Franja, Una Ruta” (овоR, por sus siglas en inglés), en 2015, comenzó a identificarse como "Belt and Road Initiative" (Iniciativa de la Franja y la Ruta) en un documento oficial 
publicado por la Comisión Nacional de Desarrollo y Reforma (Vision and Actions on Jointly Building Silk Road Economic Belt and 21stCentury Maritime Silk Road, 2015).

La "franja" está diseñada para conectar a China con Europa a través de Asia central y Rusia; al Medio Oriente a través de Asia central; y al sudeste de Asia, el sur de Asia y el océano Índico. La "ruta", busca conectar China con Europa a través del mar de China meridional y el océano Índico; y el Pacífico sur a través del mar de China meridional (Alon, Zhang y Lattemann, 2018). Se diseñaron seis corredores económicos para cumplir con estos objetivos de conectividad. Siete años después del lanzamiento oficial, la Iniciativa ya superó el ámbito territorial original, convirtiéndose en una empresa global.

La Nueva Ruta de la Seda es una estrategia económica y política con un enfoque en la inversión en infraestructura diseñada para "mejorar la conectividad física a lo largo de una ruta de la seda marítima y continental" (Mayer, 2018, p. 1228). Según Nolan (2019, p. xxi), Xi Jinping ha hecho de la nueva Ruta de la Seda "una parte clave de las relaciones internacionales de China".

Desde su inicio, la Iniciativa ha generado una gran discusión académica. Las motivaciones chinas detrás del BRI se han convertido en una preocupación central para los estudios en todo el mundo, que van desde una interpretación puramente económica hasta un enfoque geopolítico y estratégico. De acuerdo con los primeros, BRI se centra en apuntalar una economía abierta y mejorar el desarrollo interno de China, además de fomentar la cooperación internacional (Zhou, 2019; Zou, 2018). Esta perspectiva está en línea con las declaraciones oficiales sobre el propósito y los objetivos de la Iniciativa. En mayo de 2017, el pcch publicó un documento de orientación en el que afirmaba que la Iniciativa de la Franja y la Ruta "es una propuesta china cuyo objetivo es promover la cooperación pacífica y el desarrollo común en todo el mundo" (Office of the Leading Group for the Belt and Road Initiative, 2017). Los líderes del partido, y en particular $\mathrm{Xi} \mathrm{Jinping,} \mathrm{también} \mathrm{han} \mathrm{destacado} \mathrm{que} \mathrm{BRI} \mathrm{es}$ parte fundamental en el camino hacia la consecución del "sueño chino", entendido como la construcción de una sociedad moderadamente acomodada, un país rico y poderoso y un pueblo dinámico y feliz (Parra Pérez, 2017; Johnson, 2016).

Una de las motivaciones centrales de BRI se relaciona directamente con los objetivos, condiciones y demandas de la economía doméstica. Algunos de los intereses que impulsan el plan son la necesidad de consumir el exceso de capacidad industrial de China, expandir o encontrar nuevos mercados para las exportaciones, la necesidad de asegurar el acceso a los recursos naturales y encontrar nuevos usos para el excedente (Clarke, 2018; Yu, 2018). Como señala Zhou (2019), BRI complementa la reestructuración económica de China y viceversa. La Iniciativa ayuda a transformar y mejorar la industria manufacturera y a aliviar el problema del exceso de capacidad en las industrias tradicionales chinas, lo que aumenta la demanda de materiales de construcción (Johnson, 2016) y suministros industriales de alta tecnología para proyectos de infraestructura en el extranjero.

Continuando con el punto de vista interno, BRI es una herramienta que responde a ob- 
jetivos económicos y políticos, vinculados a la estabilidad y mantenimiento de la legitimidad del Partido. Esta legitimación está anclada en la mejora continua de las condiciones socioeconómicas de la población, que se convirtió en un mayor desafío para el gobierno en un contexto de desaceleración del ritmo de crecimiento exponencial de las décadas previas (Garlick, 2019). En relación con este punto, Xiaotong y Keith (2019) plantean que la diplomacia económica de Xi, está diseñada para superar el miedo a la inseguridad, vinculado al suministro de energía, la seguridad alimentaria y una mayor capacidad para hacer frente a posibles shocks económicos y financieros en la economía mundial, y a una forma de responder a los crecientes sentimientos nacionalistas en China a través de la demostración de logros económicos sostenidos.

A su vez, el desarrollo de las provincias del oeste de China, más atrasadas en comparación con las industrializadas zonas costeras, también se reconoce como uno de los objetivos internos detrás de la implementación de BRI (Ferdinand, 2016). En este sentido, BRI es una estrategia para estimular desde el Estado la redistribución de beneficios económicos hacia las zonas periféricas del país, favoreciendo a esa población a través de inversiones masivas. Se articula así el desarrollo de esas zonas con su rol como puerta de acceso al mayor mercado de consumidores del mundo (Clarke, Sussex, Bisley, 2020).

Pero además de la centralidad de los aspectos económicos y políticos domésticos del plan, BRI también refleja intenciones y preocupaciones estratégicas y geopolíticas relacionadas con el papel de China como potencia mundial (Alon, Zhang y Lattemann, 2018), combinadas con la necesidad de mantener la estabilidad regional. De acuerdo con esta afirmación, una visión común sugiere que la Iniciativa es de hecho una gran estrategia. En esta línea, Rolland (2018) sostiene que es un instrumento al servicio de la visión que la República Popular China tiene de sí misma como la potencia líder indiscutible en la región en las próximas décadas.

Clarke (2018) también agrega que "BRI es un intento de resolver los dilemas geopolíticos que surgen de la orientación estratégica 'híbrida' de China [marítima y continental]; y como respuesta a las oportunidades y desafíos presentados por el 'pivote hacia Asia' de Estados Unidos desde 2011”. Como explican Clarke et al. (2020), la dependencia China sobre las zonas costeras está vinculada a que desde allí ingresa la mayor parte de los suministros y la presencia naval de Estados Unidos y su marcada superioridad sobre la fuerza naval China, pone a Beijing frente a la necesidad de encontrar corredores alternativos de abastecimiento y así reducir la vulnerabilidad frente a un potencial corte de rutas marítimas. La BRI funciona de esta manera como una herramienta para establecer esos corredores de abastecimiento terrestres que puedan dar una mayor capacidad de resiliencia a China ante una crisis marítima.

Desde una perspectiva de diplomacia económica, que articula a la BRI como una herramienta para objetivos de índole político, se pueden también interpretar las motivaciones de Beijing en Asia central, vinculadas directamente con el temor a la inestabilidad en las regiones del oeste de China. Como sostienen 
Kastner, Pearson y Rector (2020), BRI puede facilitar el desarrollo económico de la región incrementando las conexiones internas y externas de Xinjiang a través de proyectos de infraestructura que pasen por la zona. Esto se vincula también con potenciar el desarrollo económico de los países de Asia central, que permitan disminuir la irradiación e influencia del extremismo hacia la zona occidental de China. Finalmente, la BRI puede convertirse en un incentivo para los países socios, una " $\mathrm{za}$ nahoria financiera”, que incline a los gobiernos a una mayor cooperación con Beijing, y que permita mejorar la imagen del país a nivel internacional principalmente en sus vecinos más próximos (Xue, 2019).

La BRI actúa, entonces, como un "paraguas" donde confluyen intereses nacionales externos (seguridad marítima, balancear la supremacía de Estados Unidos en la región, estabilidad regional, entre otras), así como aquellos de índole interna (seguridad alimentaria y energética, superar el desigual crecimiento entre regiones, el exceso de capacidad industrial, mantener la legitimidad del partido, etc.), haciendo de la diplomacia económica una herramienta clave para la concreción de los intereses estratégicos de Beijing.

\section{DIPLOMACIA ECONÓMICA, BRI Y EL SUR GLOBAL}

El cambio de siglo, caracterizado por la consolidación de la globalización, implicó el auge de procesos como transnacionalización, multilateralismo y diversificación de la agenda internacional. En este contexto es que surge el concepto de Sur Global, donde ya no so- lo se hace referencia a las relaciones entre los Estados del Sur, sino que se incluyen los vínculos entre actores no gubernamentales, como organizaciones, movimientos sociales, etc. (Lechini, 2013).

Cada vez son mayores los cuestionamientos acerca de las formas de entender y explicar categorías de análisis como poder, hegemonía e incluso las dinámicas Norte/Sur. Diferentes autores destacan la necesidad de ampliar las miradas, diversificar los enfoques y perspectivas, y diseñar nuevos conceptos y teorías que se adapten al nuevo contexto internacional y que se alejen del tradicional centro hegemónico de la disciplina (Lechini y Rubiolo, 2020), entre ellos es posible destacar Arlene Tickner (2016), Andrew Hurrell (2016); Amitav Acharya (2015), Amado Cervo (2008).

El Sur Global al que hacemos referencia, no es un todo homogéneo. En efecto, es posible identificar tres grandes grupos, conforme a su nivel de desarrollo socioeconómico: las economías emergentes, aquellas moderadamente desarrolladas y las economías menos desarrolladas (Zhou, 2019).

En el caso de China, que se autodefine como país en desarrollo, se observa una distancia cada vez mayor en sus capacidades materiales, no solo respecto al resto de los países del Sur Global sino, también, de los emergentes: a fines de 2001 ingresó a la Organización Mundial del Comercio (marcando un punto de inflexión en la integración china en el orden económico global). Más tarde, en 2007, se convirtió en el mayor exportador mundial y en el segundo importador y se consagrará como segunda economía global (Li, 2017). En 2008, la crisis financiera internacional se tradujo a un 
repliegue de las grandes potencias occidentales a la vez que contribuyó a fortalecer la imagen de China como potencia global. Mientras el mundo experimentaba caídas en el producto de un $0,8 \%$ y un 3,2\%, respectivamente, China logró crecer a un 8,7\% (Cepal, 2010). Esto fue posible gracias a un gigantesco paquete de estímulo económico, acompañado de una gran expansión crediticia (Busilli, 2020). De este modo, las políticas comerciales y financieras de China actualmente impactan en el resto del mundo, convirtiéndola en una especie de "vecino" para los países del globo (Li, 2017).

A partir de 2010, China reforzó su going global strategy (que incentiva a las empresas nacionales chinas a salir al exterior en busca de oportunidades de inversión) enfocándose en la inversión de empresas chinas en recursos energéticos y materias primas para abastecer a un modelo económico industrial basado en la inversión y orientado a las exportaciones. Más recientemente, la inversión se orienta a proyectos que le permitan a China ascender en las cadenas globales (Johnson, 2016; Wang, 2016). En el informe presentado ante el XIx Congreso Nacional del Partido Comunista de China [1], llevado a cabo en octubre de 2017, Xi Jinping (2017) destacó el papel prioritario de la BRI, al impulsar por igual las estrategias del bringing in y going global. En este sentido, la iniciativa se articula con la estrategia de internacionalización de las oportunidades de inversión, a través de los corredores económicos y de la ruta marítima, donde los países en desarrollo han tenido un lugar protagónico como receptores de los flujos de inversión.

De acuerdo con el relevamiento de fuentes oficiales (Green BRI Center, 2020 y Portal de la Franja y la Ruta, 2020) y artículos especializados (Serrano Moreno; Telias y Urdínez, 2020), el listado de países que firmaron mous de acceso a BRI son los que figuran en la tabla 1 .

Como puede observarse en la tabla 1 , de los 138 miembros oficiales de BRI, más del $70 \%$ son países en vías de desarrollo. Asimismo, se registra un incremento en las adhesiones durante los últimos tres años, en especial aquellas provenientes del continente africano y de la región latinoamericana (AEI, 2020). Pese a ciertas reticencias y reservas iniciales, el Sur Global se muestra cada vez más receptivo a la iniciativa: las regiones que mayores flujos de inversión han recibido desde el lanzamiento de la iniciativa han sido Asia oriental (26\%), Asia occidental (21\%), África subsahariana (20\%) y África del norte y Medio Oriente (15\%), respectivamente (Green BRI Center, 2020).

\subsection{BRI y las entidades financieras nacionales e internacionales}

Un elemento de especial importancia para comprender la relación entre diplomacia económica y la Franja y la Ruta es la financiación de los proyectos. Como se dijo antes, BRI representa el instrumento de política económica e influencia global más relevante para Beijing. De este modo, el gobierno chino financia la iniciativa a través de múltiples instituciones.

Entre ellas se destacan el Fondo de la Ruta de la Seda, que se concentra en la financiación de proyectos de inversión en infraestructura del sector energético. El Fondo se encuentra respaldado por dos importantes instituciones estatales: el Export-Import Bank of China (Eximbank), y el Chinese Development Bank 
Tabla 1

Países miembros de BRI, por regiones

\begin{tabular}{|l|c|}
\hline REGIONES & NO. PAíSES \\
TOTAL GLOBAL & 38 \\
\hline $\begin{array}{l}\text { África Subsahariana } \\
\text { Angola, Benin, Burundi, Cabo Verde, Camerún, Chad, Comoras, República del Congo, Costa de Marfil, Guinea } \\
\text { Ecuatorial, Etiopía, Gabón, Gambia, Ghana, Guinea, Kenia, Lesoto, Liberia, Madagascar, Mali, Mauritania, Mo- } \\
\text { zambique, Namibia, Níger, Nigeria, Ruanda, Senegal, Seychelles, Sierra Leona, Somalia, Sudáfrica, Sudán del } \\
\text { Sur, Sudán, Tanzania, Togo, Uganda, Zambia, Zimbabue. }\end{array}$ & 38 \\
\hline $\begin{array}{l}\text { Sur de Asia } \\
\text { Afganistán, Bangladesh, Maldivas, Nepal, Pakistán, Sri Lanka. }\end{array}$ & 6 \\
\hline $\begin{array}{l}\text { Europa y Asia Central } \\
\text { Albania, Armenia, Austria, Azerbaiyán, Bielorrusia, Bosnia y Herzegovina, Bulgaria, Croacia, Chipre, República } \\
\text { Checa, Estonia, Georgia, Grecia, Hungría, Italia, Kazajstán, Kirguistán, Letonia, Lituania, Luxemburgo, Mol- } \\
\text { davia, Montenegro, Macedonia, Polonia, Portugal, Rumania, Rusia, Serbia, Eslovaquia, Eslovenia, Tayikistán, } \\
\text { Turquía, Ucrania, Uzbekistán. }\end{array}$ & 34 \\
\hline $\begin{array}{l}\text { Este de Asia y Pacífico } \\
\text { Brunei, Camboya, Fiji, Indonesia, Islas Cook, Kiribati, República de Corea, Laos, Malasia, Micronesia, Mongo- } \\
\text { lia, Myanmar, Nueva Zelanda, Niue, Papúa Nueva Guinea, Filipinas, Samoa, Singapur, Isla Salomón, Tailandia, } \\
\text { Timor Oriental, Tonga, Vanuatu, Vietnam. }\end{array}$ & 24 \\
\hline $\begin{array}{l}\text { Medio Oriente y África del Norte } \\
\text { Argelia, Bahréin, Djibouti, Egipto, Emiratos Árabes Unidos, Irán, Irak, Kuwait, Líbano, Libia, Malta, Marruecos, } \\
\text { Omán, Qatar, Arabia Saudí, Tunisia, Yemen. }\end{array}$ & 17 \\
\hline $\begin{array}{l}\text { América Latina y el Caribe } \\
\text { Antigua y Barbuda, Barbados, Bolivia, Chile, Costa Rica, Cuba, Dominica, Ecuador, El Salvador, Granada, Guya- } \\
\text { na, Jamaica, Panamá, Perú, República Dominicana, Surinam, Trinidad y Tobago, Uruguay y Venezuela. }\end{array}$ & 19 \\
\hline
\end{tabular}

Fuente: Elaboración propia con base en datos de Green BRI Center (2020); Serrano Moreno, Telias y Urdínez (2020) y Portal de la Franja y la Ruta (2020).

(CDB). Asimismo, cuenta con el apoyo del International Finance Corporation (IFC), que representa el brazo del sector privado del Grupo del Banco Mundial.

Por otra parte, se encuentran entidades financieras multilaterales como el New Development Bank (NDB), también conocido como el banco de los Brics, que ofrece préstamos en infraestructura (del sector de energía, sobre todo a proyectos de energía renovable) a sus países miembros. Esta entidad está destinada a fortalecer la cooperación entre los Brics y complementar los esfuerzos de las instituciones financieras multilaterales y regionales para el desarrollo global (Ocde, 2018; Johnson, 2016).

El Bank of China es una de las entidades financieras estatales que participan de la Franja y la Ruta, y brinda préstamos no concesionales en proyectos relacionados con la iniciativa, así como el China Construction Bank. Otra de las instituciones es China Export and Credit Insurance Corporation (Sinosure), que en 2015 firmó un acuerdo con Industrial and Commercial Bank of China, para participar en proyectos a lo largo de la Ruta de la Seda, en materia de indemnizaciones (Ocde, 2018). 
Por último, una de las entidades financieras multilaterales más relevantes, en el marco de la iniciativa, es el Banco Asiático de Inversión e Infraestructura (AIIB). Por sus objetivos y alcance, el AIIB ha generado un importante debate. Algunos autores sostienen que China planea apropiarse del Sur Global a través de instituciones alternativas a las de Bretton Woods (Ramonet, 2015; Medeiros, 2019) y hay quienes afirman que China busca moldear el orden internacional vigente conforme a sus necesidades e intereses (Slipak y Ghiotto, 2019; $\mathrm{Fu}, 2019)$. Lo cierto es que en el AIIB China cuenta con el $26,6 \%$ de los votos, manteniendo una distancia significativa con el resto de los socios con mayor participación, como India $(7,6 \%)$ o Rusia (6\%), así como de países desarrollados occidentales que lo integran ${ }^{2}$ (AIIB, 2020). Sin dudas, esta asimetría en favor de China es considerable, y permite a Beijing asegurar que sus decisiones (y, en consecuencia, sus intereses estratégicos) se concreten (Busilli, 2020). Sin embargo, muchos de los proyectos aprobados por el AIIB son también financiados por entidades multilaterales como el Banco Mundial, el Asian Development Bank (ADB) o el Banco Europeo para la Reconstrucción y Desarrollo (BERD) $)^{3}$.

Es posible notar el predominio de las instituciones estatales chinas en la financiación, diseño y direccionamiento de los proyectos en el marco de la BRI. No obstante, también se observa una importante participación de las entidades financieras multilaterales más relevantes del sistema internacional, reflejando el interés de principales potencias occidentales en la iniciativa (a excepción de Estados Unidos), así como su proyección global. A modo de síntesis, se ofrece la siguiente tabla:

\section{Tabla 2}

\section{Entidades financieras que participan} de la Franja y la Ruta

\begin{tabular}{|c|c|}
\hline $\begin{array}{l}\text { INSTITUCIONES } \\
\text { FINANCIERAS CHINAS } \\
\text { (ESTATALES) }\end{array}$ & $\begin{array}{l}\text { INSTITUCIONES } \\
\text { FINANCIERAS } \\
\text { MULTILATERALES }\end{array}$ \\
\hline Eximbank & $\begin{array}{l}\text { Banco Asiático de Inversión } \\
\text { e Infraestructura (AllB) * }\end{array}$ \\
\hline $\begin{array}{l}\text { Chinese Development Bank } \\
\text { (CDB) }\end{array}$ & $\begin{array}{l}\text { New Development Bank } \\
\text { (NDB)* }\end{array}$ \\
\hline Bank of China & $\begin{array}{l}\text { Asian Development Bank } \\
\text { (ADB) }\end{array}$ \\
\hline China Construction Bank & $\begin{array}{l}\text { International Finance Corpo- } \\
\text { ration (IFC) }\end{array}$ \\
\hline Sinosure & Banco Mundial \\
\hline $\begin{array}{l}\text { Industrial and Commercial } \\
\text { Bank of China }\end{array}$ & $\begin{array}{l}\text { Banco Europeo para la } \\
\text { Reconstrucción y Desarrollo } \\
\text { (BERD) }\end{array}$ \\
\hline
\end{tabular}

Fuente: Elaboración propia con base en datos provistos por Ocde (2018), Johnson (2016) y documentos oficiales (Memorándum de Entendimiento sobre Cooperación en Materia de Intereses Comunes en el Marco de la Iniciativa de la Franja y de la Ruta, 2017).

* Bancos multilaterales liderados por China.

2 Entre ellos Austria, Bélgica, Francia, Alemania, Grecia, Italia, Países Bajos, entre otros. Para conocer la lista completa de países miembros del AIIB, consultar: https://www.aiib.org/en/about-aiib/governance/members-of-bank/index.html

3 Para un análisis más detallado sobre las instituciones que colaboran en la Franja y la Ruta, consultar documento completo del Memorándum de Entendimiento sobre Cooperación en Materia de Intereses Comunes en el marco de la Iniciativa de la Franja y de la Ruta, 14 de mayo de 2017. Recuperado de https://www.ndb.int/wp-content/ uploads/2018/09/MOU-on-BRI-signed.pdf 


\subsection{Las inversiones en el Sur global:} destinos, proyectos e influencia

En este apartado nos concentramos en las inversiones programadas y concretadas dentro del marco de la BRI a los principales destinos con foco en los países en desarrollo. Como puede observarse en el gráfico 1, los sectores receptores de mayores flujos de inversión china han sido: energía (39\% del total), transporte (25\%), bienes raíces $(10 \%)$ y metales $(8 \%)$ (AEI, 2020). En este sentido, la preeminencia de estas áreas de inversión se relaciona directamente con los objetivos de asegurar el acceso a recursos naturales y fuente de energía, reutilizar el excedente de capacidad productiva china y acceso a nuevos mercados, tal como se plantea en el apartado 2.

Respecto a las regiones, en el período 2013-2020, Asia oriental ha sido la mayor destinataria de los flujos de inversión, por valor de 196 mil millones de dólares. Le sigue Asia occidental, con 161,2 mil millones; África subsahariana, con 154 mil millones; Medio Oriente y el norte de África, con 110,8 miles de millones; Europa, con 76 mil millones y, por último, América Latina y el Caribe con 49,5 mil millones de dólares (AEI, 2020).

Conforme al objeto de estudio del trabajo, se tomará el sector de energía y transporte, dado que representan los mayores flujos de inversión durante el período seleccionado y,

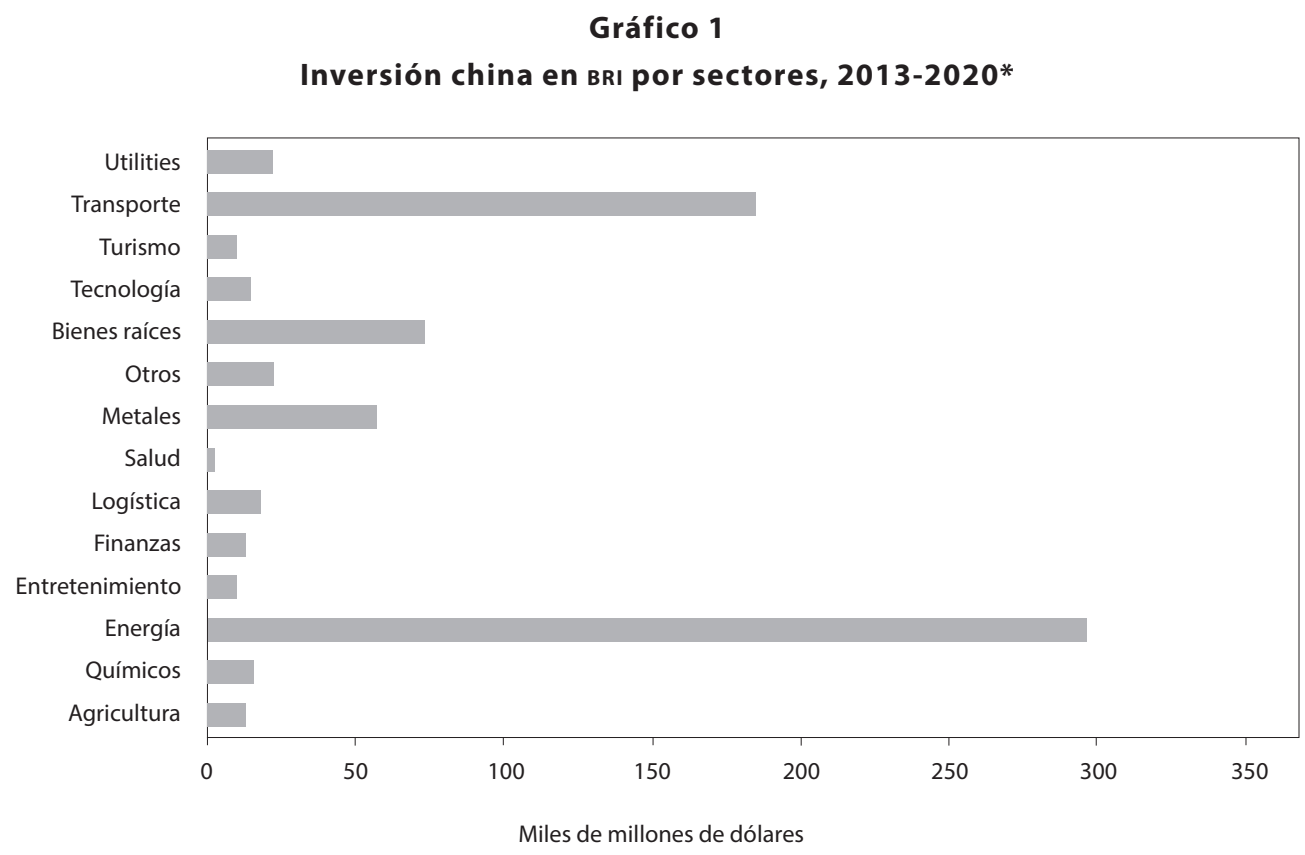

Fuente: Elaboración propia con base en American Enterprise Institute (AEI) China Global Tracker, 2020.

*Los valores correspondientes a 2020 pertenecen al primer semestre del ańo. 


\section{Gráfico 2}

Principales países receptores de los flujos de inversión china BRI. Sector energético, 20132020*. En miles de millones de dólares.

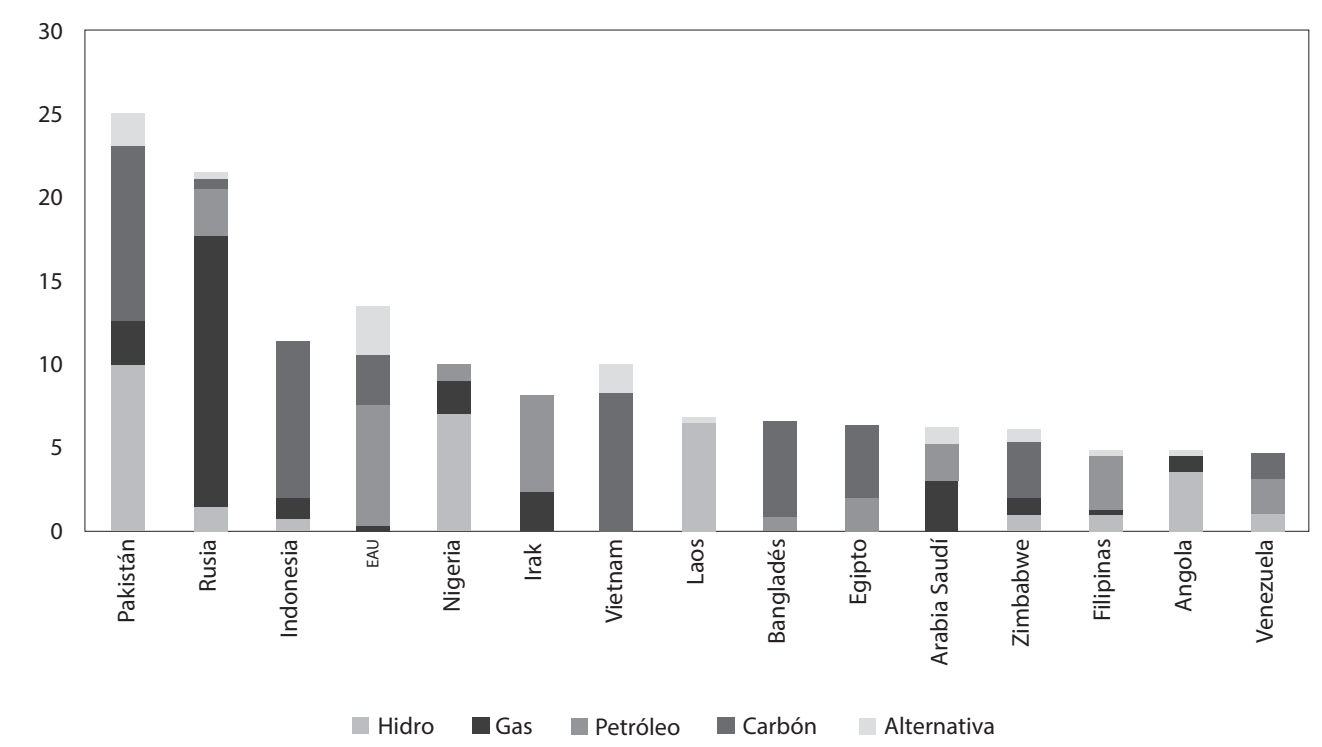

Fuente: Elaboración con base en American Enterprise Institute (AEI) China Investment Tracker (2020) y Green Belt and Road Initiative Center (2020).

*Los valores correspondientes a 2020 pertenecen al primer semestre del año.

en conjunto, superan el $60 \%$ de la inversión total (470 mil millones aproximadamente, de los 755 mil millones de dólares invertidos hasta el momento) (AEI, 2020).

El gráfico 2 muestra los quince países destinatarios de los principales flujos de inversión, durante el período analizado. Conforme a esta información puede observarse que, a excepción de Rusia ${ }^{4}$, los destinos de inversión más importantes corresponden a países del Sur Global.

Es posible observar también la prioridad de los flujos de inversión hacia la periferia de

4 Rusia es el segundo mayor receptor de flujos de inversión y forma parte del Corredor Económico China-MongoliaRusia. Si bien los proyectos previstos para este corredor corresponden a varios sectores, como comercio, infraestructura, se vuelve estratégico para China porque permite garantizar su seguridad energética, principalmente de gas, que captó 16,2 mil millones de dólares durante el período analizado. Una de las inversiones principales en gas natural licuado es el Proyecto Yamal LNG, ubicado en la península rusa de Yamal en el Ártico. La planta alcanzó su capacidad de producción total, con tres líneas de producción, cada una de las cuales tiene una capacidad de 5,5 millones de toneladas por año (Spanish People Daily, 08/04/2019). 
China, donde concentra importantes intereses estratégicos, como fuentes de abastecimiento, seguridad energética y proyección de liderazgo regional, dado que la presencia e influencia en Asia resulta fundamental para consolidar su ascenso en el sistema internacional (Bhattacharya, 2016; Delage, 2015, Callahan, 2016). En este sentido, en la región del sur de Asia se destacan las inversiones de Pakistán (25,1 mil millones de dólares) y Bangladesh (6,6 mil millones de dólares) que concentran proyectos relacionados con los subsectores de carbón, gas y energía hidroeléctrica, principalmente (Green BRI Center, 2020). En el caso de Pakistán, que forma parte del Corredor Económico China-Pakistán, ocupa el primer lugar como destino de inversión. Se destacan proyectos como la planta hidroeléctrica de Dasu Hidropower, ubicada a pocos kilómetros de la ciudad Dasu (Pakistán) que generará energía limpia, capaz de generar 2,16 GW. Respecto a la energía a base de carbón, se destaca la Planta de Sahiwal (provincia de Punjab), operativa desde 2017. Con un monto de inversión de 1,9 mil millones de dólares aproximadamente, fue llevado a cabo por las empresas chinas Huaneng Shandong y Shandong Ruyi Technology Group. La contraparte pakistaní Punjab Power Development Board (РPDB) fue la agencia de supervisión (Power Technology, 2019).

En la región del este de Asia y el Pacífico, se destacan las inversiones en Indonesia (11,5 mil millones), Vietnam (10,1 mil millones), Laos (6,8 mil millones) y Filipinas (4,9 mil millones), en proyectos relacionados con los subsectores del carbón, petróleo y energía hidroeléctrica principalmente (Green BRI
Center, 2020). Todos ellos forman parte del Corredor Económico China-Península de Indochina. En el caso de Indonesia, los principales flujos de inversión se concentran en el subsector del carbón (9,4 mil millones de dólares), donde se destaca la construcción de la planta de carbón por la empresa china Shenhua Energy Company en conjunto con la empresa local Perusahaan Listrik Negara (PLN), a $100 \mathrm{~km}$ de la capital, Jakarta (China Shenhua Energy Company Limited, 2015).

En la región de Medio Oriente y África del norte se encuentran proyectos relacionados con los subsectores de petróleo, gas y carbón, principalmente. Se destacan Emiratos Árabes Unidos (13,6 mil millones), Irak (8,2 mil millones), Egipto (6,4 mil millones) y Arabia Saudí (6,2 mil millones) como principales destinos de inversión (AEI, 2020). En el caso de Emiratos Árabes Unidos, entre 2017 y 2018, la empresa estatal China National Petroleum Corporation adquirió acciones en los campos petrolíferos de la compañía petrolera más grande del país, Abu Dhabi National Oil Company (Adnoc), por valor de casi 3 mil millones de dólares (World Energy Trade, 2020).

En la región de África Subsahariana priman las inversiones en los subsectores de carbón y energía hidroeléctrica, donde Nigeria (10 mil millones), Zimbabwe (6,1 mil millones) y Angola (4,8 mil millones) captaron los mayores flujos de inversión durante el período seleccionado. Por último, la región de América Latina y el Caribe, donde se destacan las inversiones realizadas en Venezuela, por valor de 4,7 mil millones de dólares, que se dirigieron a proyectos relacionados con el petróleo, la energía hidroeléctrica y el carbón. 
Principales países receptores de los flujos de inversión china en BRI. Sector de transporte 2013-2019. En miles de millones de dólares americanos.

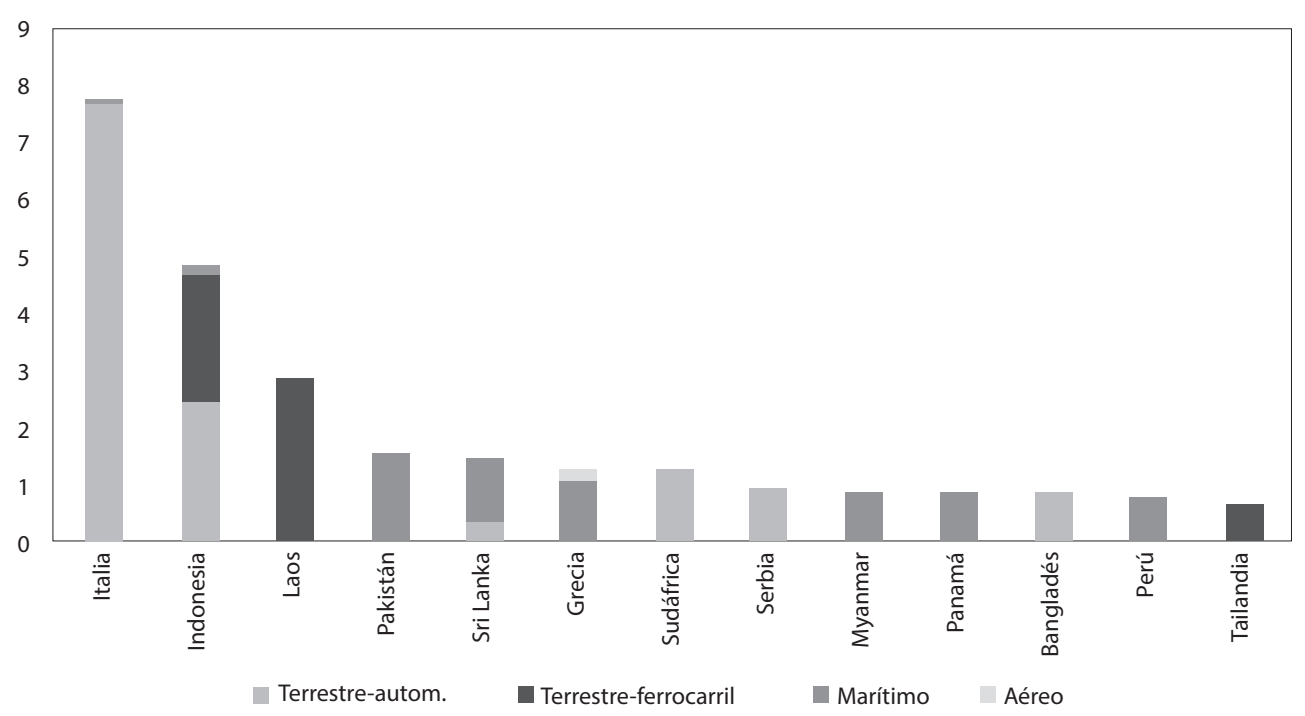

Fuente: Elaboración con base en American Enterprise Institute (AEI) China Investment Tracker (2020) y Green Belt and Road Initiative Center (2020).

*Los valores correspondientes a 2020 pertenecen al primer semestre del año.

A modo de resumen, las cifras muestran que, a través de los diferentes corredores económicos, China refuerza su seguridad energética a partir de empresas estatales (o vinculadas al Estado), fortaleciendo los flujos de inversión, diversificando sus socios comerciales e impulsando a sus empresas a la acción internacional. Y para ello, la Franja y la Ruta juega un rol estratégico en la diplomacia económica que China lleva adelante bajo el liderazgo de Xi Jinping.

El gráfico 3 muestra una tendencia similar a la anterior: de los trece mayores destinos de inversión, tan solo Italia y Grecia ${ }^{6}$ pertenecen al mundo desarrollado. Los once países restantes, forman parte del Sur Global.

5 La inversión registrada hace referencia a la adquisición de la empresa de neumáticos Pirelli, por la China National Chemical Corp (ChemChina), por valor de 7,7 miles de millones en 2015 (Reuters, 2015).

6 En 2019 la empresa estatal china Cosco Shipping realizó una inversión de 670 millones de dólares en el puerto más grande de Grecia, el Pireo, para impulsar su papel como centro en el rápido crecimiento del comercio entre Asia y Europa, transformándolo en el puerto más grande de Europa (Reuters, 2019). 
A nivel regional, el este de Asia y el Pacífico recibieron los mayores flujos de inversión. Indonesia (4,9 mil millones de dólares) y Laos (2,9 mil millones de dólares) se ubican en el segundo y tercer puestos como destinos de inversión. Con mayor distancia se ubican Myanmar (900 millones de dólares) y Tailandia (700 millones de dólares). En Indonesia los capitales se concentraron en el transporte terrestre: carreteras (2,5 miles de millones de dólares) y ferrocarril (2,2 miles de millones de dólares). Respecto a este último, uno de los proyectos ferroviarios más importantes es el tren de alta velocidad que une Yakarta y Bandung, en Indonesia. La inversión fue realizada por China Railway Group Limited (Crec), por un valor de 2,2 mil millones de dólares (Global Times, 2020; AEI, 2020). En transporte de automóviles destaca la inversión de la empresa China Communications Construction Co, quien compró la participación PT Jasamarga Probolinggo Banyuwangi, por un valor de 1,6 miles de millones de dólares, para construir una carretera de peaje de $170 \mathrm{~km}$, que conectará la ciudad de Probolinggo con Banyuwangi en la costa Este de la isla principal de Java (Reuters, 2019).

En el caso de Laos, la inversión se concentró en el transporte terrestre ferrocarril (2,9 mil millones de dólares). En 2018 la empresa China Railway Engineering, China Railway Corporation realizó una inversión de 2,9 miles de millones de dólares para la construcción del ferrocarril transfronterizo (Xinhua, 2020).

El sur de Asia se ubica como otro importante destino. En él se destacan las inversiones realizadas en Pakistán (1,6 mil millones de dólares), Sri Lanka (1,5 mil millones de dólares) y Bangladés (900 millones de dólares). En el caso de Pakistán se destaca la inversión de 1,6 mil millones de dólares por la empresa estatal China Overseas Port Holding Co (que opera el puerto de Gwadar en Pakistán), al contratar a la empresa de infraestructura portuaria Zhuhai Port Holdings Group Co Ltd, para la labor conjunta en el marco de BRI. El proyecto no solo impulsará el desarrollo económico en la provincia donde se encuentra el puerto de Gwadar, sino que también conectará la región autónoma Uygur de Xinjiang y otras provincias occidentales de China con Sri Lanka, Bangladés, Omán, Emiratos Árabes Unidos, Irán e Irak (China Daily, 2015).

En Europa y Asia Central priman las inversiones realizadas en Serbia (mil millones). Se destaca la inversión de la empresa china Shandong, con 994,4 millones de dólares en la construcción de una fábrica de neumáticos en la Zona Franca de Zrenjanin de Serbia (Xinhua, 2019).

En América Latina y el Caribe se destacan las inversiones en países como Panamá (900 millones de dólares) y Perú (800 millones de dólares). En ambos casos los mayores se relacionan al transporte marítimo. En Panamá, el grupo chino de logística y energía, Landbridge Group, invirtió 900 millones de dólares aproximadamente, para desarrollar en nuevo puerto de aguas profundas en la Isla Margarita de Panamá, en la entrada atlántica del Canal de Panamá (Infrastructure Investor, 2016).

Por último, la región de África subsahariana, donde los flujos de inversión en Sudáfrica fueron los más elevados, por valor de 1,3 mil millones de dólares. Relacionadas a proyectos de transporte terrestre de carreteras se destaca 
la inversión de la empresa estatal china, Beijing Automobile International Corporation (Baic), en una zona industrial en la provincia de Eastern Cape de Sudáfrica, para la construcción de una planta de fabricación de automóviles (Reuters, 2016).

A modo de cierre, las inversiones chinas en el Sur Global se concentran en infraestructura $y$, en particular, en dos grandes regiones: el sudeste de Asia y el sur de Asia. En ambos espacios, los principales receptores son economías de desarrollo medio y bajo. Excede el alcance del artículo cubrir los flujos de IED en cada país que forma parte de la Iniciativa, pero es ilustrativo observar la participación de la IED china sobre el total en algunos de los países que se constituyen, de acuerdo con lo analizado previamente, en focos de los corredores que integran la BRI. En la tabla 3 se muestran los datos de IED para 2019 en países seleccionados.

A partir de estos datos puede observarse la relevancia del financiamiento en el total de las inversiones de los países asiáticos que re- presentan puntos estratégicos en la diplomacia económica china. En Pakistán, Laos y Camboya, la participación de China muestra una relevancia superlativa. El incremento de la participación equivale a una mayor dependencia sobre el financiamiento chino, que desde una perspectiva de diplomacia económica puede ser potencialmente explotada por Beijing para influir en el comportamiento de sus contrapartes. Los casos de Camboya y Laos en las disputas por el mar de China meridional y la presión de Beijing para evitar que ambos gobiernos apoyen la postura de Asean (Zhang, 2018) en el conflicto, son un ejemplo paradigmático del potencial uso de las herramientas económicas para fines políticos vinculados a intereses centrales de la República Popular China.

\section{CONCLUSIONES}

Desde la diplomacia económica, y más cercana a la noción de economic statecraft, Beijing procura sostener y proyectar el poder económico

Tabla 3

Inversión extranjera directa a países seleccionados de BRI, 2019 (en millones de US\$)

\begin{tabular}{|l|c|c|c|}
\hline & TOTAL DE IED & TOTAL DE IED CHINA & \% DE CHINA \\
\hline Pakistán & 2,218 & 1,387 & 62.5 \\
\hline Indonesia & 24,946 & 4,744 & 19.0 \\
\hline Laos & $2,321^{*}$ & 1,072 & 46.2 \\
\hline Vietnam & $31,800^{* *}$ & 3,560 & 11.2 \\
\hline Camboya & 3,663 & 2,750 & 75.1 \\
\hline
\end{tabular}

* Hace referencia a los proyectos aprobados para inversión extranjera.

** Hace referencia a la IED comprometida.

Elaboración propia con datos de: World Bank (2021), State Bank of Pakistan (2021), Indonesia Investment Coordinating Board (2020), ocDE (2018), Laos Ministry of Planning and Investment (2021), Vietnam Ministry of Planning and Investment (2020), Council for the Development of Cambodia (2021). 
a través de la Belt and Road Initiative, con fines políticos. Por medio de una cooperación económica sin precedentes (Xiaotong y Keith, 2017), y con el foco en proyectos de infraestructura cuenta con seis corredores económicos que conectan Asia con Europa y África de manera integral y de largo plazo.

Como se sostiene a lo largo del trabajo, la BRI tiene como principal objetivo convertir la riqueza china en poder. En ella convergen y se entrelazan intereses nacionales de orden interno y externo. Entre los primeros se destacan la seguridad alimentaria y energética, el fortalecimiento de la legitimidad del Partido (a través del éxito de las reformas), y el intento por reducir las diferencias de las regiones chinas en el proceso modernizador. Los segundos se relacionan con la seguridad marítima y rutas de abastecimiento, así como al intento por consolidar su rol como potencia regional en Asia-Pacífico y proyectar su poder a escala global. De este modo, BRI se transforma en una estrategia que permite articular intereses estratégicos a través de un diseño flexible, con foco en la interconectividad de Eurasia, pero abierto al mundo a través de la Ruta de la Seda Marítima.

En línea con lo anterior y retomando a autores como Alan, Zhang y Latterman (2018), el análisis de las inversiones realizadas demuestra el carácter prioritario de los países del Sur Global como destinatarios de los mayores flujos de inversión, en especial aquellos que pertenecen a Asia. Más aún, China se ha convertido en su principal fuente de IED, reforzando las dinámicas de la diplomacia económica y fortaleciendo el papel de China en la región. Entre ellos, se destacan los proyectos de Pakistán, Indonesia y Laos, quienes forman parte de dos de los corredores económicos más importantes para Beijing y evidencian el carácter prioritario de la región como plataforma de proyección de poder regional y global (Arase, 2016).

Ahora bien, en la medida en que avanza la iniciativa surgen los cuestionamientos sobre las consecuencias que pueda tener para el Sur Global aceptar un proyecto de escala global con sello chino. En el marco de la pandemia, la BRI genera interrogantes. En primer lugar, la articulación que realizará China entre su propia recuperación económica pospandemia y la financiación e implementación de los proyectos a escala global. En este sentido, las proyecciones de crecimiento del PBI de China para 2020 se sitúa en 1,2\% (lejos del 6.1\% estimado a principios del año), pero que sin dudas la ubica por delante del resto del mundo, que registraría tasas negativas (FMI, 2020).

Finalmente, la BRI como herramienta de economic statecraft está en función no solo del grado de participación financiera de China en el país receptor, sino también de variables como la proximidad geográfica y la existencia de intereses centrales -en particular vinculados a la integridad territorial del país-. El sudeste de Asia, el sur de Asia y Asia central son espacios estratégicos dentro del Sur Global, mientras que América Latina tiene un espacio de relevancia periférica. Por ello, es probable que en los próximos años se observe una intensificación de las inversiones -acompañadas de un mayor flujo de créditos y ayudas-y de la utilización de incentivos para ejercer influencia en el comportamiento de los Estados comprendidos en esas zonas neurálgicas. 
La BRI representa oportunidad y riesgo para el mundo menos desarrollado, y permite una diversificación de socios, pero refuerza la asimetría y la dependencia sobre grandes poderes (Rubiolo, 2020). En este sentido, la activa diplomacia económica china, está creando también las condiciones para que la asimetría pueda ser potencialmente aprovechada en procura de los intereses políticos de Beijing.

\section{REFERENCIAS}

Acharya, A. (2015). An IR for the Global South or a Global IR? Indonesian Journal of International Studies (IIIS). Recuperado de https://www.e-ir. info/2015/10/21/an-ir-for-the-global-south-ora-global-ir/

American Enterprise Institute. (2020). Disponible en https://www.aei.org/

Amin, A. y Thrift, N. (1997). Globalization, socio-economics, territoriality. En: R. Lee y J. Wills (eds.), Geographies of economies. Londres: Routledge, pp. 147-157.

Alon, I.; Zhang, W. y Lattemann, C. (2018). China's Belt and Road Initiative: Changing the Rules of Globalization. Switzerland: Palgrave Macmillan.

Asian Infrastructure Investment Bank (2020). Disponible en https://www.aiib.org/en/index.html

Arase, D. (2016). The geopolitics of Xi Jinping's Chinese dream: problems and prospects. Iseas-Yusof Ishak Institute.

Baldwin, R. (1985). Economic Statecraft. Princeton University Press: New Jersey.

Bhattacharya, A. (2016).Conceptualizing the Silk Road Initiative in China's Periphery Policy. East Asia 33, 309-328.

Bayne, N.; Woolcock, S. (2018). What is Economic Diplomacy?, en N. Bayne y S. Woolcock (eds.),
The new economic diplomacy. Decision making and negotiation in international economic relations. London: Routledge: 3-20.

Berridge, G. R. y James, A. (2003). A Dictionary of Diplomacy. Basingstoke: Palgrave.

Blanchard, J-M. F. y Ripsman, N. M. (2008). A Political Theory of Economic Statecraft, Foreign Policy Analysis 4: 371-398.

Busilli, V. (2020). Belt and Road Initiative (BRI): la iniciativa estratégica de Xi Jinping. Cuadernos de Politica Exterior Argentina (Nueva Época), 131: 69-88.

Callahan, W. (2016). Chinas "Asia Dream.” Asian Journal of Comparative Politics, 1(3): 226-243.

Cervo, A. (2008). Inserção Internacional. Formação dos conceitos brasileiros. São Paulo: Editora Saraiva.

China Daily. (2015). Zhuhai Port scores big with deal in Pakistan. Disponible en http://usa.chinadaily. com.cn/epaper/2015-10/30/content_22326165. htm

China Shenhua Energy Company Limited. (2015). Overseas Regulatory Announcement, Beijing. Disponible en http://en.shenhuachina.com.cn/ uploadfiles/shenhua_china_en/1452848759287. pdf

Clarke, M. (2018). The Belt and Road Initiative: Exploring Beijing's Motivations and Challenges for its New Silk Road. Strategic Analysis, 42(2): 84-102.

Clarke, M.; Sussex, M. y Bisley, N. (2020). The Belt and Road Initiative and the Future of Regional Order in the Indo-Pacific. London: Lexington Books.

Coolsaet, R. (2004). Trade and Diplomacy: The Belgian Case, International Studies Perspectives, 5(1): 61-65.

Council for the Development of Cambodia (2021). FDI Trend. Disponible en: http://www.cambodiainvestment.gov.kh/why-invest-in-cambodia/ investment -enviroment/fdi-trend.html 
Delage, F. (2015). La estrategia asiática de Xi Jinping. Revista del Instituto Español de Estudios Estratégicos, $5,1-36$.

Ferchen, M. (2018). How New and Crafty is China's "New Economic Statecraft"? Carnegie-Tsinghua Center for Global Policy Department of International Relations, Tsinghua University.

Ferdinand, P. (2016). Westward ho-the China dream and 'one belt, one road': Chinese foreign policy under Xi Jinping, Internationl Affairs 92: 941957.

Fondo Monetario Internacional. (10/2020). Perspectivas de la Economía Mundial. Disponible en https://www.imf.org/es/Publications/weo/Issues/2020/09/30/world-economic-outlook-october-2020

Fu, M. (2019). The Belt and Road Initiative from Three Theoretical Perspectives. CICIR, 1-33. Disponible en http://www.cicir.ac.cn/UpFiles/file/20200227/6371841699726745496853430. pdf

Garlick, J. (2020). The Impact of China's Belt and Road Initiative. From Asia to Europe. New York: Routledge.

Gilpin, R. (2001). Global Political Economy. Understanding the International Economic Order. Princeton: Princeton University Press.

Global Times. (2019). El proyecto del tren bala YakartaBandung impulsa la economía local y aporta transferencias de tecnología. Disponible en https://www.globaltimes.cn/content/1159479. shtml

Green Belt and Road Center. (2020). Disponible en https://green-bri.org/about-us

Haan, Arjan de. (2011). Development Cooperation as Economic Diplomacy. The Hague Journal of Diplomacy 6 (1-2): 203-217.
Hill, C. (2016). Foreign policy in the twenty-first century. Londres: Pallgrave Macmillan.

Hufbauer, G.C.; Schott, J. y Elliott, K. (1990). Economic Sanctions Reconsidered: History and current policy. Washington: Institute for International Economics

Hurrell, A. (2016). Towards the Global Study of International Relations, Revista Brasileira de Politica Internacional 59(2): 1-18.

Indonesia investment coordinating board. (2020). Domestic and foreign direct investment realization. Quarter IV and January - December 2019 Disponible en: https://www.bkpm.go.id/images/ uploads/file_siaran_pers/Paparan_Bahasa_Inggris_Press_Release_Tw_IV_2019.pdf

Infrastructure Investor. (2016). Landbridge Group de China adquiere puerto de Panamá. Disponible en https://www.infrastructureinvestor.com/chinaslandbridge-group-acquires-panama-port/

Johnson, C. (2016). President Xi Jinping's Belt and Road Initiative. A Practical Assessment of the Chinese Communist Party's Roadmap for China’s Global Resurgence. Center for Strategic and International Studies, Washington DC, pp. 1-38.

Karsten, S.; Pearson, M.; Rector, C. (2020). China's strategic multilateralism. Investing in global governance. Cambridge: Cambridge University Press.

Laos Ministry of Planning and Investment (2021). Investment Promotion Department. Statistics. Disponible en: http://investlaos.gov.la/resources/ statistics/

Lechini, G. (2013). Reflexiones en torno a la Cooperación Sur-Sur. Escenarios XXI, 16: 1-12.

Lechini, G. y Rubiolo, F. (2020). 100 años de relaciones internacionales. Miradas desde el Sur Global. Studia Politice, 50: 5-10.

Li, Y. (2017). Belt and Road: A Logic Behind the Myth. En Amighini, A. (Ed.). China's Belt and Road: a 
Game Changer? Milano, Italia: Edizioni Epoké, 13-34.

Mastanduno, M. (1998). Economics and security in statecraft and scholarship, International Organization 52 (4): 825-854.

Ministry of Commerce People's Republic of China. (2020). Annual Statistical Communiqué of China’s Outward Foreign Direct Investment Statistical Year Book. Disponible en http://english. mofcom.gov.cn/article/newsrelease/significantnews/202009/20200903004178.shtml

National Development and Reform Commission. (2015). Vision and Actions on Jointly Building Silk Road Economic Belt and 21stCentury Maritime Silk Road. Ministry of Foreign Affairs, and Ministry of Commerce of the People's Republic of China. Disponible en http://www.beltandroadforum.org/english/n100/2017/0410/c22-45.html.

Norris, W. J. (2010). Economic statecraft with Chinese characteristics: the use of commercial actors in China's grand strategy (Doctoral dissertation, Massachusetts Institute of Technology). Disponible en https://dspace.mit.edu/handle/1721.1/62474

Ocde. (2018). China's Belt and Road Initiative in the Global Trade, Investment and Finance Landscape. Business and Finance Outlook, 1-46.

Okano-Heijmans, M. (2011). Conceptualizing Economic Diplomacy: The Crossroads of International Relations, Economics, IPE and Diplomatic Studies, The Hague Journal of Diplomacy 6: 7-36.

Okano-Heijmans, M. (2013). Economic Diplomacy: Japan and the Balance of National Interests. Leiden: Brill.

Parra Pérez, A. (2017). Obor: las 5 claves de la mayor iniciativa de infraestructuras mundial liderada por China, Documento Opinión 113, Instituto Español de Estudios Estratégicos: 1-20.
Parra Pérez, A. (2020). ¿Retos pospandemia?: China pide paso. Documento Opinión 80, Instituto Español de Estudios Estratégicos: 1-15.

Portal de la Franja y de la Ruta. Disponible en www. yidaiyilu.gov.cn

Power Technology. (2019). Port Qasim Coal-Fired Power Plant, Karachi. Disponible en https://www. power-technology.com/projects/port-qasim-coalfired-power-plant-karachi/

Rabby, F. (2015). Small states in international political economy (IPE): Challenging the challenges, Foreign Affairs Insights \& Review. Disponible en https://fairbd.net/small-states-in-international-political-economy-ipe-challenging-thechallenges/

Rana, K. (2013). Economic diplomacy: what might best serve a developing country? International Journal of Diplomacy and Economy, 1(3/4), 232.

Reuters. (2016). Beijing Automobile Intl Corp to invest $\$ 800 \mathrm{mln}$ in S.African industrial zone. Disponible en https://www.reuters.com/article/ozabs-ussafrica-china-investment-idAFKCN10U0NH

Reuters. (2019). China Communications Construction unit to invest $\$ 1.65$ billion in Indonesian toll road. Disponible en https://www.reuters.com/ article/us-indonesia-china-communications/ china-communications-construction-unit-toinvest-1-65-billion-in-indonesian-toll-roadidUSKBN1WT1I5

Rubiolo, F. (2020). The South China Sea Dispute: A Reflection of Southeast Asia's Economic and Strategic Dilemmas (2009-2018), Revista de Relaciones Internacionales, Estrategia y Seguridad, 15 (2): 115-130.

Rubiolo, F.; Busilli V. y Escobar, M. (2020). Análisis de la política exterior de Xi Jinping hacia el sudeste de Asia: Estrategias, intereses y dimensiones. Revista Relaciones Internacionales, 93 (2): 83-118. 
Scholvin, S.; Wigell, M. (2019). Geo-economic power politics. An introduction. En M. Wigell, S. Scholvin y M. Aaltola (eds.), Geo-Economics and Power Politics in the 21st Century. The Revival of Economic Statecraft. New York: Routledge: 1-13.

Serrano Moreno, J.; Telias, D. y Urdínez, F. (2020). Deconstructing the Belt and Road Initiative in Latin America. Asian Education and Development Studies. Disponible en https://www.emerald.com/insight/content/doi/10.1108/AEDS-01-2020-0021/ full/html?skipTracking=true

Slipak, A. y Ghiotto L. (2019). América Latina en la Nueva Ruta de la Seda. El rol de las inversiones chinas en la región en un contexto de disputa (inter)hegemónica. Cuadernos del CEL. 4 (7): 26-55.

State Bank of Pakistan. (2021). Net Inflow of Foreign Private Investment (Archive). Disponible en: https:// www.sbp.org.pk/ecodata/NIFP_Arch/index.asp

Stopford, J.; Strange, S. (1991). Rival States, Rival Firms. Competition for world market shares. London: University Press, Cambridge.

Strange, S. (1990). Finance, information and power, Review of International Studies, 16(03), 259.

Strange, S. (1992). States, Firms and Diplomacy, International Affairs 68 (1): 1-15.

Tickner, J. A. (2016). Knowledge Is Power: Challenging IR's Eurocentric Narrative, International Studies Review: 1-2.

Tussie, D. (2015). Relaciones internacionales y economía política internacional: Notas para el debate. Relaciones Internacionales, 48: 155-175.

Vietnam Ministry of Planning and Investment. (2020). Socioeconomic information statistics. Disponible en: http://www.mpi.gov.vn/en/Pages/ktxh. aspx?idcm $=289$

Wang, J. (2019). The six economic corridors. En Fang, C. \& Nolan, P. (ed.), Routledge Handbook of the
Belt and Road, Routledge International Handbooks, 195-223.

World Bank. (2021). Foreign direct investment, net inflows statistics. Disponible en: https://data. worldbank.org/indicator/BX.KLT.DINV.CD.WD

World Energy Trade. (2020). Por primera vez una compañía petrolera offshore de China se une a las concesiones de Adnoc. Disponible en https:// www.worldenergytrade.com/finanzas-energia/ economia/por-primera-vez-una-compania-petrolera-offshore-de-china-se-une-a-las-concesionesde-adnoc

Xi, J. (18/10/2017). Secure a Decisive Victory in Building a Moderately Prosperous Society in All Respects and Strive for the Great Success of Socialism with Chinese Characteristics for a New Era. xIx National Congress of the Communist Party of China (CPC). Beijing. Disponible en http:// www.xinhuanet.com/english/special/201711/03/c_136725942.htm

Xiaotong, Z. y Keith, J. (2017). From Wealth to Power: China's New Economic Statecraft, The Washington Quarterly, 40(1): 185-203.

Xinhua. (2019). Chinese tire maker starts building factory in Serbia. Disponible en http://www.xinhuanet.com/english/2019-03/30/c_137936846.htm

Xinhua. (2020). China-Laos railway drills through last major tunnel. Disponible en http://www.xinhuanet.com/english/2020-09/22/c_139387502.htm

Xue, G. (2019). China's Economic Inducement towards Vietnam: What Lies Ahead? En M. Li (ed.), China's Economic Statecraft: Co-optation, Cooperation, and Coercion. Singapore: World Scientific Publishing, 65-93.

Yu, J. (2018). The belt and road initiative: domestic interests, bureaucratic politics and the EU-China relations, Asia Europe Journal 16: 223-236. 
Zhang, D. (2018). The Concept of 'Community of Common Destiny' in China's Diplomacy: Meaning, Motives and Implications, Asia \& the Pacific Policy Studies, 5 (2): 196-207.

Zhang, F. (2012). Rethinking China's grand strategy: Beijing's evolving national interests and strategic ideas in the reform era. International Politics, 49(3), 318-345.
Zhou, F. (2019). China's economic restructuring and the Belt and Road. En A, Fang y P. Nolan (eds.), Routledge Handbook of the Belt and Road, New York: Routledge: 15-20.

Zou, L. (2018). The Political Economy of China's Belt and Road Initiative. Singapore: World Scientific Publishing. 